\title{
Efecto de la atorvastatina en la expresión de Fas (CD95/Apo-1) en tejido cerebral normal e isquémico
}

\author{
C. ORIA DE SUÁREZ1', S. SUÁREZ RODRÍGUEZ', L. PONCE HERNÁNDEZ², \\ J. CORADO RAMÍREZ ${ }^{2}$, R. TOVAR MENDOZA², A. EBLEN-ZAJJURZ
}

${ }^{1}$ Laboratorio de Fisiopatología Experimental (FIPEX). ${ }^{2}$ Unidad de Investigaciones en Inmunología (UNIVENIN). ${ }^{3}$ Laboratorio de Neurofisiología. Facultad de Ciencias de la Salud. Universidad de Carabobo. Valencia. Venezuela

\author{
EFFECT OF ATORVASTATIN ON FAS (CD95/APO-1) EXPRESSION \\ IN NORMAL OR ISCHEMIC BRAIN
}

\section{RESUMEN}

Introducción: La fisiopatología de la isquemia cerebral involucra múltiples eventos, entre ellos, importantes mecanismos apoptóticos donde la proteína Fas es expresada. Las estatinas poseen efectos neuroprotectores independientes de sus acciones hipolipemiantes.

Objetivo: Estudiar el efecto de la atorvastatina sobre la expresión de Fas en tejido cerebral isquémico.

Material y métodos: Quince ratas Sprague Dawley, machos, fueron distribuidas en tres grupos $(n=5)$ : un grupo control sin isquemia cerebral y dos experimentales ( 1 y 2 ) sometidos a isquemia cerebral global inducida por paro respiratorio con D-tubocurarina. El grupo experimental 2 recibió atorvastatina durante las dos semanas previas a la inducción de isquemia $(10 \mathrm{mg} / \mathrm{kg} / \mathrm{día}$, v.o.). Se realizó inmunohistoquímica por el sistema Avidina-Biotina-Peroxidasa a muestras de la corteza cerebral frontal.

Resultados: Fas se expresó en el 38\% de las células del tejido no isquémico. Esta expresión aumentó en el tejido cerebral isquémico a $63 \%(\mathrm{P}=0,0003)$. La atorvastatina inhibió significativamente la expresión de Fas en tejido cerebral isquémico en un 23\% (P = 0,0007).

Conclusiones: La atorvastatina disminuyó la expresión de Fas en isquemia cerebral, contribuyendo posiblemente a una inhibición de la apoptosis. Este mecanismo de acción pudiera explicar parte de su efecto neuroprotector. Estos hallazgos sugieren que la atorvastatina pudiera resultar beneficiosa en la prevención primaria de los ataques isquémicos.

PALABRAS CLAVE: Apoptosis. Atorvastatina. Fas (CD95/Apo-1). Isquemia cerebral. Muerte neuronal. Neuroprotección.

\section{ABSTRACT}

Background: The physiopathology of the brain ischemia includes many events such as important apoptotic mechanisms were Fas protein is expressed. Statins have neuroprotector effects that are independent of their blood lipid reducing actions.

Objective: Study of the atorvastatin effect on Fas expression in brain ischemia.

Material and methods: Fifteen male Sprague Dawley rats were distributed in three groups $(n=5)$, the control group without cerebral ischemia, in the other two experimental groups (1 and 2) a global cerebral ischemia were induced by respiratory arrest (D-tubocurarin). Experimental group 2 was treated with atorvastatin $(10 \mathrm{mg} / \mathrm{Kg} / \mathrm{d}$, p.o. $)$ during two weeks before the induction of the ischemia. Inmunohistochemical study of brain frontal specimens was done by the AvidinBiotin-Peroxidase method.

Results: Fas was expressed in 38\% of cells in non ischemic brain, this expression increased to $63 \%$ of cells in inchemic brain samples $(P=0.0003)$ Fas expression in ischemia was significantly inhibited by atorvastatin in $23 \%(P=0.0007)$

Conclusions: Atorvastatin decreased Fas expression in ischemic brain. This fact must be taken into account to explain the atorvastatin neuroprotection and suggests the potential use such as primary prevention for ischemic attacks.

KEY WORDS: Apoptosis. Atorvastatin. Brain ischemia. Fas (CD95/Apo1). Neuronal death. Neuroprotection.

Oria de Suárez, C, Suárez Rodríguez, S, Ponce Hernández, L, Corado Ramírez. J, Tovar Mendoza R, Eblen-Zajjur A. Efecto de la atorvastatina en la expresión de Fas (CD95/Apo-1) en tejido cerebral normal e isquémico. An Med Interna (Madrid) 2006; 23: 156-160.

\section{INTRODUCCIÓN}

Los ataques isquémicos cerebrales (AIC), constituyen un importante problema de salud pública, en los que es difícil ejercer una acción terapéutica profiláctica, salvo en aquellos casos donde el paciente haya presentado, de inicio, un ataque isquémico transitorio o tenga factores predisponentes, como, hipertensión arterial, diabetes mellitus, entre otros (1).
En la isquemia cerebral, la muerte de las neuronas puede ocurrir por necrosis o por apoptosis $(2,3])$. La muerte neuronal inducida por isquemia es un proceso dinámico, resultado de la activación de una cascada de eventos bioquímicos que incluyen, entre otros: alteración de la expresión de genes proapoptóticos, activación de proteasas de cisteína (caspasas) y endonucleasas que llevan a la degradación final del núcleo $(4,5)$. 
Estudios previos plantean que la apoptosis juega un papel importante en la fisiopatología de la isquemia cerebral focal (6), e igualmente, que las señales de muerte mediadas por Fas (receptor de membrana implicado en muerte celular por apoptosis), intervienen en la muerte neuronal después de una isquemia cerebral global $(3,7)$. Se ha demostrado, en estos casos, la activación de las vías intrínseca y extrínseca de la apoptosis mediada por caspasas, así como la activación de la vía de señalización independiente de caspasas (8). Por otra parte, Ferrer y Planas (2003) demostraron un incremento de la expresión de Fas y FasL después de una isquemia cerebral focal, así como aumento de la expresión de caspasas 1, 3, 8 y 9 y del clivaje de la caspasa 8 , en el área de la penumbra isquémica.

Basándose en estos conocimientos, se ha desarrollado una variedad de estrategias terapéuticas neuroprotectoras como el uso de inhibidores de las caspasas, proteínas antiapoptóticas virales, modulación de la expresión de proteínas anti y proapoptóticas sistémicas y antagonistas de receptores de muerte $(9,10)$. El uso de estas drogas de reciente investigación, sin embargo, no muestra resultados muy esperanzadores para prevenir la isquemia cerebral (4).

Investigaciones realizadas en ratones con deficiencia de Fas, han demostrado que tienen tendencia a hacer menos isquemia (3). Esto hace pensar que si un medicamento es capaz de reducir la expresión de Fas, también pudiera ser capaz de reducir la aparición de la isquemia.

Las estatinas son capaces de reducir el riesgo de ataques isquémicos en sujetos con altos niveles de colesterol $(11,12,13)$. Por otra parte, se ha demostrado que poseen efectos pleiotrópicos, relacionados principalmente, con la inhibición de la HMG-CoA reductasa $(14,15)$. Adicionalmente, muestran un efecto neuroprotector independiente de su acción hipolipemiante, atribuido a sus propiedades antiinflamatorias y antioxidantes (16), reduciendo el tamaño del infarto cerebral (11).

A fin de ahondar en el estudio de los mecanismos neuroprotectores de la atorvastatina, este estudio se propuso investigar: 1) la expresión del receptor de membrana que desencadena señales de muerte apoptótica Fas (CD95) en condiciones de isquemia cerebral global, y 2) el efecto de la Atorvastatina (inhibidor de la HMG CoA reductasa) sobre la expresión de Fas (CD95) en el modelo experimental de isquemia cerebral global en ratas.

\section{MATERIAL Y MÉTODOS}

Quince (15) ratas Sprague Dawley, machos, con peso promedio de 320 gr; fueron distribuidas en tres grupos, un grupo control $(\mathrm{n}=5)$ y dos grupos experimentales $(\mathrm{n}=5 ; \mathrm{c} / \mathrm{u})$, sometidas a las mismas condiciones de ambiente y de alimentación, ritmo de luz:oscuridad 12:12 horas, comida y agua $\mathrm{ad}$ libitum. Los grupos experimentales fueron sometidos a isquemia cerebral global, mientras que el grupo control fue sometido a la misma manipulación pero sin producir la isquemia. Las ratas del grupo 2 recibieron, además, $10 \mathrm{mg} \cdot \mathrm{kg}^{-1}$.día ${ }^{-1} \mathrm{de}$ Atorvastatina (Parke-Davis ${ }^{\circledR}$ ) por vía oral durante dos semanas (17). Los procedimientos experimentales se realizaron de acuerdo a lo indicado por el National Institute of Health y la U.S. Public Health Office sobre el uso de animales de experimentación (18) y fueron avalados por la Dirección de Investi- gación y Producción Intelectual de la Facultad de la Salud.

Los animales se anestesiaron con tiopental sódico $(60$ mg. $\mathrm{kg}^{-1}$ i.p.), una vez verificada la profundidad de la anestesia por ausencia de reflejo de retiro, se procedió de la siguiente manera: A las ratas de los grupos experimentales 1 y 2 se les indujo isquemia cerebral global por hipoxia debida a paro respiratorio al administrar D-tubocurarina (0,2 mg.kg-1, i.p.) siguiendo metodología reportada con anterioridad (19). Se vigiló cuidadosamente el momento en que ocurrió el paro respiratorio y a los 10 minutos, se extrajo rápidamente una muestra de corteza cerebral del lóbulo frontal izquierdo de aproximadamente $1 \mathrm{~mm}^{3}$. Las ratas del grupo control (no sometidas a isquemia), se les aplicó el mismo procedimiento de obtención de tejido cerebral en los mismos tiempos descritos.

\section{ESTUDIO DE LA EXPRESIÓN DE FAS POR INMUNOHISTOQUÍMICA}

Se realizaron cortes de $5 \mu \mathrm{m}$ de espesor, colocándose en láminas portaobjetos tratadas con poli-L-lisina y se sometieron a tinción con hematoxilina-eosina y a la técnica de Avidina-Biotina-Peroxidasa por el método indirecto. Las muestras fueron hidratadas con solución amortiguadora de fosfato (PBS) y secuencialmente incubadas en cámara húmeda a temperatura ambiente, en diferentes etapas con: 1) anticuerpo monoclonal primario anti CD95 (R\&D Systems ${ }^{\circledR}$ ) a una dilución de 1/1000 durante 40 minutos; 2) anticuerpo secundario biotinado antiinmunoglobulina de rata (BRAR) generado en conejo (Vector $\operatorname{Labs}^{\circledR}$ ) a una dilución de $1 / 30$ por 30 minutos, y 3) sistema revelador enzimático con solución Cromógeno "Novared" (Vector Labs ${ }^{\circledR}$ ) por 4 minutos. Los cortes fueron lavados con agua corriente y contrastados con Hematoxilina Gill'S (Vector Labs ${ }^{\circledR}$ ) por 5 minutos. Entre cada incubación se realizó un lavado con PBS durante 5 minutos. Las láminas fueron sometidas luego, a deshidratación por pasos sucesivos de 3 minutos con alcohol $70 \%$, alcohol $100 \%$, y a dos pasos sucesivos en xilol $100 \%$, para permitir la preservación de la reacción inmunológica en el tejido utilizando medio de montaje Entellan permanente hidrofóbico $\left(\mathrm{Merck}^{\circledR}\right)$. Se realizaron controles negativos en cada experimento en los cuales no se añadió el anticuerpo primario y en su lugar se colocó PBS. Las láminas fueron procesadas el mismo día, por tres investigadores de manera, coordinada, independiente y simultánea, para detener la reacción enzimática al mismo tiempo.

Las láminas se observaron al microscopio óptico. Se consideró como positiva de la expresión de CD95, cuando las membranas celulares y el tejido tomaron una coloración pardo rojiza. Se consideró expresión negativa cuando el corte tomó una coloración violeta. Los cortes fueron fotografiados con una cámara digital con una resolución de 4,8 Megapixels. Las imágenes se procesaron digitalmente con el programa Imagetool 3.0 con el cual se realizó el contaje de células. La observación y análisis de los cortes fue realizado por tres investigadores de forma independiente.

\section{ANÁLISIS DENSITOMÉTRICO DE LA EXPRESIÓN DE FAS}

La lectura densitométrica fue realizada con el programa Imagetool 3.0. Para ello las imágenes coloreadas fueron previamente convertidas a una escala de grises, de tal manera que las áreas con mayor expresión de Fas mostraban una mayor densidad óptica expresada en unidades densitométricas. Estos 
datos se trasladaron a un programa estadístico (Statistica 6.0) para establecer la base de datos, realizar cálculos y analizar los resultados.

\section{ANÁLISIS ESTADÍSTICO}

El contaje de células fue expresado en frecuencia relativa (\%). Los valores densitométricos fueron presentados como media aritmética \pm error estándar (EE). Se determinó el tipo de distribución de los datos mediante el test de Kolmogorov Smirnov, como la misma no era gaussiana, se procedió al análisis de los datos utilizando el test de Friedman. Se consideraron significativas las diferencias cuando $\mathrm{p}<0,05$.

\section{RESULTADOS}

Expresión de Fas en tejido cerebral normal e isquémico. En la Tabla I se observa la comparación de la expresión celular cortical de Fas en el grupo de ratas sin isquemia cerebral y en el grupo de ratas con isquemia cerebral global. Se muestra que Fas se expresa en los dos grupos, sin embargo, dicha expresión fue significativamente mayor $(p=0,0003)$ en el grupo con isquemia que en el grupo sin isquemia cerebral (63 vs. $38 \%)$

\section{TABLA I}

EXPRESIÓN DE Fas EN TEJIDO CEREBRAL NORMAL (GRUPO CONTROL) E ISQUÉMICO (GRUPO 1)

\begin{tabular}{cl}
\hline$\%$ DE CÉLULAS & \\
\hline Positivas & Negativas \\
\hline
\end{tabular}

\begin{tabular}{lcc}
$\begin{array}{l}\text { Grupo control } \\
\text { (sin Isquemia) }\end{array}$ & 38 & 62 \\
$\begin{array}{l}\text { Grupo 1 } \\
\text { (con Isquemia) }\end{array}$ & 63 & 37 \\
\hline
\end{tabular}

Control vs. Grupo $1(p=0,0003)$

\section{EXPRESIÓN DE Fas EN TEJIDO CEREBRAL ISQUÉMICO DE RATAS} SIN TRATAMIENTO Y TRATADAS CON ATORVASTATINA

La evaluación del perfil densitométrico del tejido cerebral del grupo control y del isquémico (Grupo 1), se muestra en la Figura 1, observándose que en el grupo con isquemia, los valores densitométricos fueron significativamente mayores, en los perfiles dorso-ventrales, que en el grupo control ( $p=0,00000)$ (Figs. 2 y 3).

En la Tabla II se observa la expresión celular de Fas en el tejido cerebral isquémico de las ratas. El grupo de ratas que recibió atorvastatina, expresó significativamente mayor Fas que el grupo isquémico que no la recibió $(\mathrm{p}=0,0007)$.

El análisis del perfil densitométrico dorso-ventral para los Grupos 1 y 2 mostró igualmente valores mayores para el grupo que no recibió atorvastatina que para el grupo tratado con atorvastatina $(\mathrm{p}=0,00000)($ Figs. 3-5).

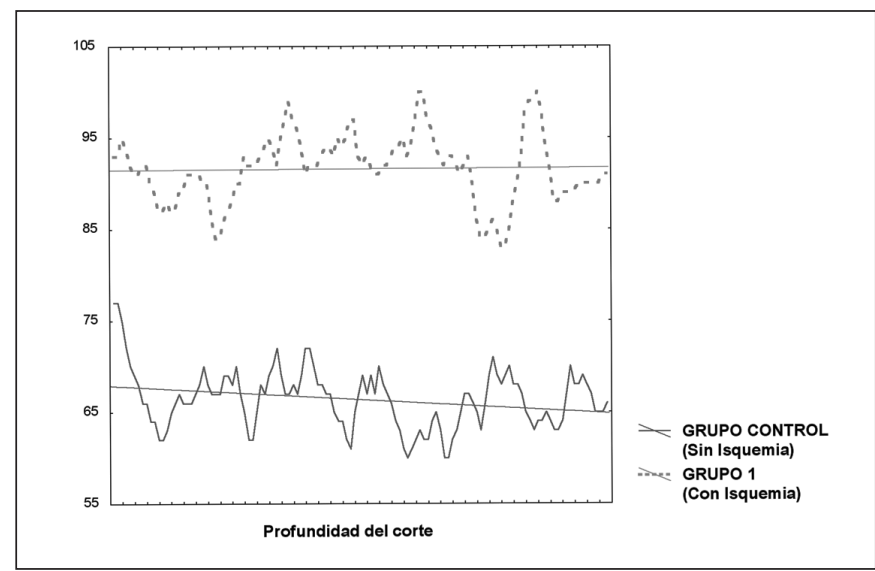

Fig. 1. Perfil densitométrico de la expresión de Fas en el tejido cerebral normal (Grupo Control) e isquémico (Grupo 1) de ratas. Distribuidos de acuerdo a Grupos de estudio y representados en unidades desintométricas.

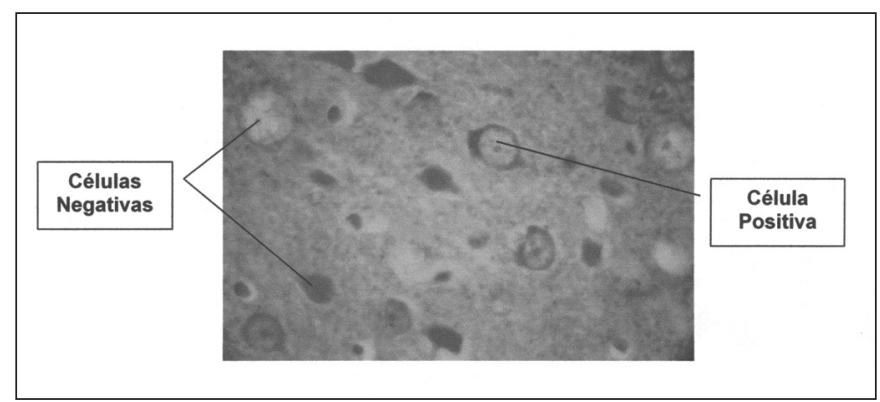

Fig. 2. Expresión de Fas (CD95) en tejido cerebral no isquémico de ratas (Grupo Control). Técnica Inmunohistoquímica de Avidina Biotina Peroxidasa, 40x.

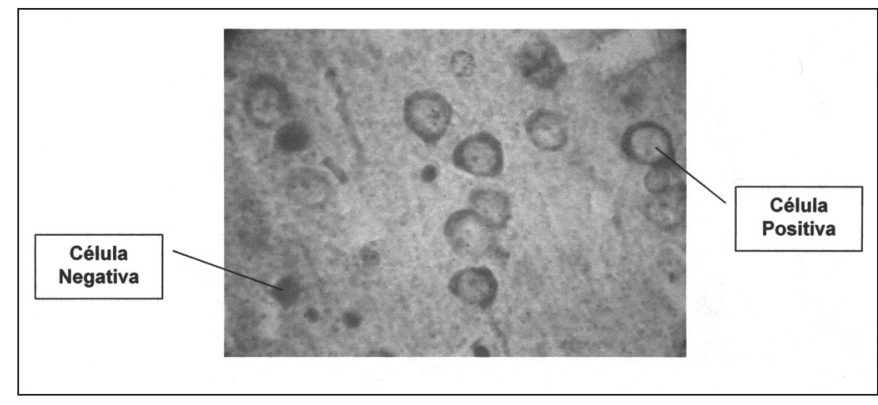

Fig. 3. Expresión de Fas (CD95) en tejido cerebral de ratas sometidas a isquemia cerebral global (Grupo 1), técnica inmunohistoquímica de Avidina Biotina Peroxidasa, 40x.

\section{DISCUSIÓN}

Entre las opciones terapéuticas para la prevención de los ataques isquémicos se han propuesto a las estatinas (20,21). La acción profiláctica de estos medicamentos es independiente de su acción hipolipemiante y más bien, debida a efectos pleiotrópicos, los cuales han sido asociados a la disminución de las consecuencias de la isquemia en modelos experimentales (22-24) e incluso a reducción del tamaño del infarto (25).

En este trabajo se investigó el efecto de la atorvastatina sobre la expresión de Fas, en tejido cerebral isquémico y sano 


\begin{tabular}{|c|c|c|}
\hline \multicolumn{3}{|c|}{ TABLA II } \\
\hline \multicolumn{3}{|c|}{$\begin{array}{c}\text { EXPRESIÓN DE Fas EN TEJIDO CEREBRAL ISQUÉMICO SIN } \\
\text { ATORVASTATINA (GRUPO 1) E ISQUÉMICO CON } \\
\text { ATORVASTATINA (GRUPO 2) }\end{array}$} \\
\hline \multicolumn{3}{|c|}{ \% DE CÉLULAS } \\
\hline & Positivas & Negativas \\
\hline $\begin{array}{l}\text { Grupo } 1 \\
\text { (Con Isquemia sin } \\
\text { atorvastatina) }\end{array}$ & 63 & 37 \\
\hline $\begin{array}{l}\text { Grupo } 2 \\
\text { (Con Isquemia con } \\
\text { atorvastatina) }\end{array}$ & 40 & 60 \\
\hline
\end{tabular}

Grupo 1 vs. Grupo 2 ( $p=0,0007)$

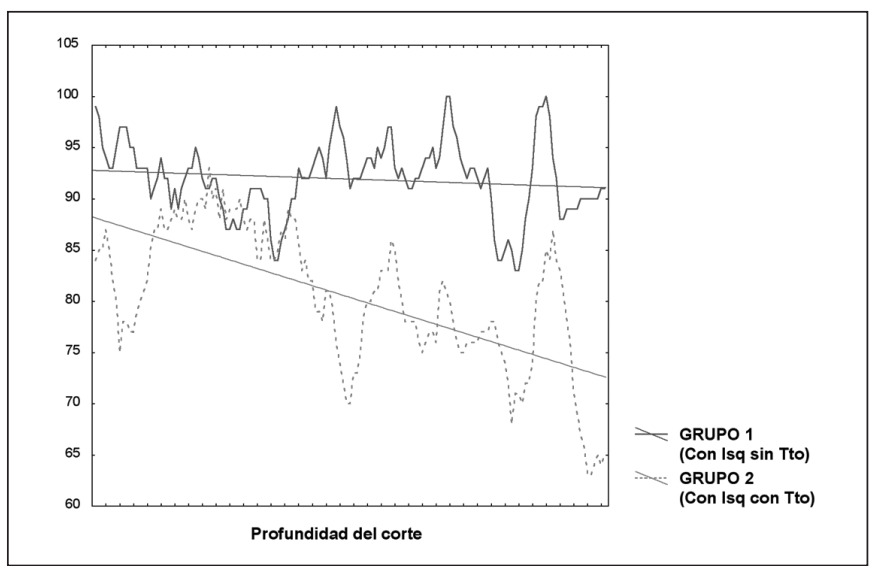

Fig. 4. Perfil densitométrico de expresión de Fas en tejido cerebral isquémico sin tratamiento (Grupo 1) e isquémico con tratamiento con atorvastatina (Grupo 2).

de ratas. Los resultados muestran una expresión basal de Fas en el tejido cerebral normal. En este sentido existe controversia entre diferentes grupos de trabajo, así, Medana y cols. (26) y Sung y cols. (27) reportan expresión de esta molécula en tejido cerebral normal y Chulhee y cols. (28) reportan que la expresión de la misma depende de la inducción precoz de TNF- $\alpha$ - e interferón $\gamma$, por lo que se expresan solamente en tejido cerebral lesionado.

Observamos además, que la expresión de Fas fue significativamente más elevada en el tejido cerebral sometido a isquemia que en tejido cerebral sano. Esto coincide con los datos aportados por Ferrer y Planas (8). Uno de los mecanismos que podría estar implicado en el aumento de la expresión de Fas en tejido cerebral isquémico sería, el de la participación conjunta de células (linfocitos, macrófagos o microglias) y citocinas (IL-1, IFN- $\gamma$ y TNF- $\alpha$ ), capaces de inducir la expresión de esta molécula durante los procesos de isquemia (28).

Reportes previos muestran que un déficit de la expresión de Fas se asocia con lesiones isquémicas menos prominentes

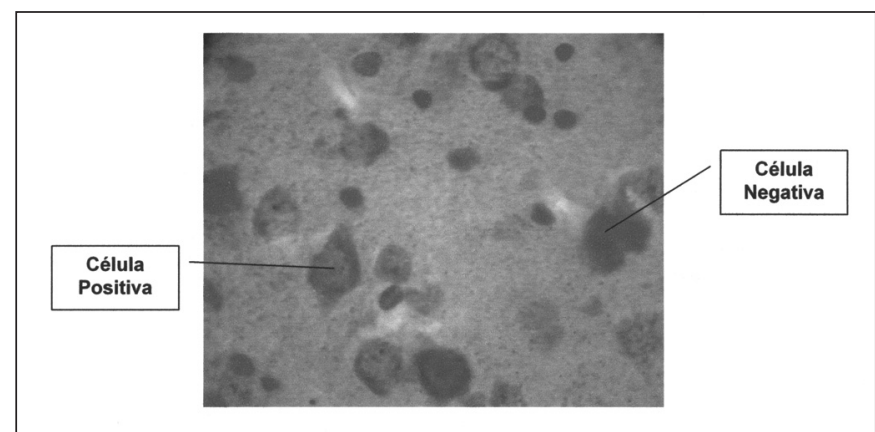

Fig. 5. Expresión de Fas (CD95) en tejido cerebral isquémico de ratas tratadas con Atorvastatina (Grupo 2), técnica Inmunohistoquímica de Avidina Biotina Peroxidasa, 40x.

en cerebro de ratones (3). Estos hallazgos sugieren que una menor expresión de la molécula de Fas, disminuye la apoptosis, con lo cual se preservaría el tejido cerebral, y que el daño tisular observado en el cerebro isquémico, se debe, al menos en parte, a la apoptosis mediada por Fas.

La atorvastatina redujo significativamente la expresión de Fas tanto en células como en parénquima cerebral. Estos resultados junto a aquellos donde la atorvastatina administrada a ratones, durante dos semanas, protege del daño ocasionado por el ataque isquémico (29), sugieren que la atorvastatina disminuye significativamente la apoptosis durante la isquemia cerebral. Por otro lado, la atorvastatina reduce el número de células apoptóticas y el daño tisular en isquemia retiniana experimental (30), así como la actividad de la caspasa 3 con disminución de la apoptosis neuronal (31).

Estudios en otros tejidos como miocitos cardiacos y células musculares lisas vasculares (32-34) muestran que la atorvastatina disminuye la apoptosis, lo que está en concordancia con el efecto observado, en el presente estudio en el tejido cerebral.

Se ha demostrado experimentalmente que las estatinas son capaces de inducir neuroprotección preventiva mediada por sus propiedades antiinflamatorias y antioxidantes $(35,36)$. Esto sugiere que muchos pacientes serían beneficiados con el uso de una terapéutica combinada que incluya el uso de estatinas en el tratamiento de los ataques isquémicos (37). Las observaciones del presente estudio sugieren fuertemente la utilidad de la atorvastatina en la reducción de la expresión de Fas asociado a la apoptosis de los ataques isquémicos.

\section{AGRADECIMIENTO}

Este trabajo fue subvencionado por el Consejo de Desarrollo Científico y Humanístico de la Universidad de Carabobo (CDCH-UC), según oficios No 209-2004 y 211-2004 de Enero de 2004.

Se agradece a todas las personas que colaboraron en la realización de este estudio, especialmente a los Técnicos de Laboratorio Olga Regalado, Luz Marina Martínez, Alexis Alvarado y María de los Ángeles Pinto, e igualmente a la Secretaria Samantha Saturno. 


\section{Bibliografía}

1. Arocha I. Lípidos, placas e hipolipemiantes. 2000. Editorial Ex Libris. ISBN 980-07-6389-9. 35-62

2. Lipton P. Ischemic cell death in brain neurons. Physiol Rev 1999; 79: 1431-1568.

3. Martín-Villalba A, Hahne M, Kieber S, Vogel J, Falk W, Schenkel J, et al. Therapeutic neutralization of CD95.ligand and TNF attenuates brain damage in stroke. Cell Death Differ 2001; 8: 679-686.

4. Hou S, MacManus J. Molecular mechanisms of cerebral ischema induced neuronal death. Int Rev Cytol 2002; 221: 93-148.

5. Leker R, Shohami E. Cerebral ischemia and trauma-different etiologies yet similar mechanisms: neuroprotective opportunities. Brain Res Rev 2002; 39: 55-73

6. Rosenbaum D, Gupta G, D'Amore J, Singh W, Zhang H, Kessier J. Fas (CD95/APO-1) plays a role in the pathophysiology of focal cerebral ischemia. J Neurosci Res 2000; 61: 686-692.

7. Jin K, Graham S, Mao X, Nagayama T, Simon R, Greenberg D. Fas (CD95) may mediate delayed cell death in hippocampal CA1 sector after global cerebral ischemia. J Cereb Blood Flow Metab 2001; 21: 1411-1421.

8. Ferrer I, Planas A. Signaling of cell death and cell survival following focal cerebral ischemia: life and death struggle in the penumbra. J Neuropathol Exp Neurol 2003; 62: 329-339.

9. Padosch S, Vogel P, Bottiger B. Neuronal apoptosis following cerebral ischemia, Basis, physiopathology and treatment strategies. Anaesthesist. 2001; 50: 905-920.

10. Padosch S, Popp E, Vogel P, Bottiger B. Altered protein expression levels of Fas/CD95 and Fas ligand in differentially vulnerable brain areas in rats after global cerebral ischemia. Neurosci Lett 2003; 338: 247-251.

11. Hess D C, Demchuk A M, Brass L M, Yatsu F M. HMG-CoA reductasa inhibitors (statins). A promising approach to stroke prevention. Neurology 2000; 54: 790-796.

12. Stalenhoef AF, Stehouwer CD. Additional effects of statins independent of the cholesterol-lowering as yet not shown to be clinically relevant. Ned Tijdschr Geneeskd 2000; 144: 308-310.

13. Gil-Núñez AC, Villanueva JA. Advantages of lipid-lowering therapy in cerebral ischemia: role of HMG-CoA reductase inhibitors. Cerebrovasc Dis 2001; 11 (Supl. 1): 85-95.

14. Farnier M. The hyperlipidemias, Role of various statins. Presse Med. 1999. 28: 2002-2010.

15. Laufs U, Liao J.K. Direct vascular effects of HMG-CoA reductasa inhibitors. Trends Cardiovasc Med 2000; 10: 143-148.

16. Vaughan CJ, Delanty N, Basson CT. Do statins afford neuroprotection in patients with cerebral ischaemia and stroke. CNS Drugs 2001. 15:589-596.

17. Laufs U, Gertz K, Huang P, Nickenig G, Bohm M, Dirnagl U, et al. Atorvastatin upregulates type III nitric oxide synthase in trombocytes, decreases platelets activation, and protects from cerebral ischemia in normocholesterolemic mice. Stroke 2000; 31: 2442-2449.

18. National Institute Of Health. Guide for the care and use of laboratory animals. NIH publications $1996 ; \mathrm{N}^{\circ} 80-23$.

19. Oria C, Eblen A. Efectos extrarrenales de la furosemida sobre compartimientos liquidos del tejido cerebral normal e Isquémico. Rev Neurol
$2001 ; 32: 414-416$

20. Alberts M. Update on the treatment and prevention of ischaemic stroke. Curr Med Res Opin 2003; 19: 438-441

21. Lalouschek W, lang W, Greisenegger S, Muliner M. Determination of lipid profiles and use of statins in patients with ischemic stroke or transient ischemic attack. Stroke 2003; 34: 105-110.

22. Bordet R, Gele P, Deplanque D, Duriez P. Antilipemics: from prevention to protection. Therapie 2003; 58: 69-76.

23. Mueck A, Seeger H. Statins and direct vascular actions. Panminerva 2003; 45: 1-6.

24. Davignon J, Mabile L. Mechanisms of action of statins and their pleiotropic effects. Ann Endocrinol 2001; 62: 101-112.

25. Kawashima S, Yamashita T, Miwa Y, Ozaki M, Namiki M, Hirase T, et al. HMG-CoA reductase inhibitor has protective effects against stroke events in stroke-prone spontaneously hypertensive rats. Stroke 2003 34: 157-163.

26. Medana I, Zhaoxia L, Alexander F, Jurg T, Hartmut W, Harald N. Fas Ligand (CD95L) Protects Neurons against perforin-mediated T lymphocyte cytotoxicity J Inmunol. 2001.167:674-681

27. Sung L, Tong Z, Chulhee Ch, Zheng W, Etty B. Differential regulation and function of Fas expression on glial cells. J Inmunol 2000; 164 1277-1285

28. Chulhee Ch, Joo Y, Jeongi L, Jung-Hee L, Eui-Cheoi S, Young A, et al Fas Ligand and Fas are expressed constitutively in human astrocytes and the expression increase with IL-1, IL-6, TNF- $\alpha$, or IFN- $\gamma$. J Inmunol 1999; 162: 1889-1895.

29. Gertz K, Laufs U, Lindauer U, Nickening G, Bohm M, Dimagi U, et al. Withdrawal of statin treatment abrogates stroke protection in mice. Stroke 2003; 34: 551-557

30. Honjo M, Tanihara H, Nishijima K, Kiryu J, Hond Y, Yue B, et al. Statin inhibits leukocyte-endothelial interaction and prevents neuronal death induced by ischemia-reperfusion injury in the rat retina. Arch ophthalmol 2002; 120: 1707-1713.

31. Focking M, Besselmann M, Trapp T. Statins potentiate caspasa-3 activity in immortalized murine neurons. Neurosci Lett 2004; 355: 41-44.

32. Tanaka K, Honda M, Takabatake T. Anti-apoptotic effect of atorvastatin, a 3-hydroxy-3-methylglutaryl coenzime A reductase inhibitor, on cardiac myocites through protein kinase $\mathrm{C}$ activation. Clin Exp Pharmacol Physiol 2004; 31: 360-364.

33. Atalar E, Ozmen F, Haznedaroglu I, Acil T, Ozer N, Ovunc K, et al Effects of short-term atorvastatin treatment on global fibrinolytic capacity, and sL-selectin and sFas levels in hyperlipidemic patients with coronary artery disease. Int J Cardiol 2002; 84: 227-231.

34. Knapp A, Huang J, Starling G, Kiener P. Inhibitors of HMG-CoA reductase sensitive human smooth muscle cells to Fas-ligand and cytokine-induced cell death. Aterosclerosis 2000; 152: 217-227.

35. Bordet R. Preventive neuroprotection: from experimental data to therapeutic strategies. Therapie 2002; 57: 540-547.

36. Vaughan CJ, Delanty N, Basson CT. Statin therapy and stroke prevention. Curr Opin Cardiol 2001; 16: 219-224.

37. Madhavan R, Chaturvedi S. Transient ischaemic attacks: new approaches to management. CNS Drugs 2003; 17: 293-305 